\title{
Whole-cell biocatalysis platform for gram-scale oxidative dearomatization of phenols
}

\author{
Summer A. Baker Dockrey ${ }^{1,2}$ \\ Tyler J. Doyon ${ }^{1,3}$ \\ Jonathan C. Perkins ${ }^{1,2}$ \\ Alison R. H. Narayan ${ }^{1,2,3}$ (D)
}

${ }^{1}$ Life Sciences Institute, University of

Michigan, Ann Arbor, Michigan

${ }^{2}$ Department of Chemistry, University of

Michigan, Ann Arbor, Michigan

${ }^{3}$ Program in Chemical Biology, University

of Michigan, Ann Arbor, Michigan

Correspondence

Alison R. H. Narayan, Life Sciences

Institute, University of Michigan, Ann

Arbor, MI.

Email: arhardin@umich.edu

Funding information

National Institute of General Medical

Sciences, Grant/Award Number: R35

GM124880

\begin{abstract}
Technologies enabling new enzyme discovery and efficient protein engineering have spurred intense interest in the development of biocatalytic reactions. In recent years, whole-cell biocatalysis has received attention as a simple, efficient, and scalable biocatalytic reaction platform. Inspired by these developments, we have established a whole-cell protocol for oxidative dearomatization of phenols using the flavindependent monooxygenase, TropB. This approach provides a scalable biocatalytic platform for accessing gram-scale quantities of chiral synthetic building blocks.
\end{abstract}

\section{K E Y W O R D S}

biocatalysis, flavin-dependent monooxygenase, oxidative dearomatization, preparative-scale, whole-cell reactions

\section{INTRODUCTION}

Growth in the areas of new enzyme discovery and development of novel biocatalytic reactions, combined with advances in protein engineering strategies, have positioned biocatalysis to have a greater footprint in synthetic chemistry (Rudroff et al., 2018; Sheldon \& Woodley, 2018). Biocatalytic methods also provide green alternatives to traditional chemical reactions by avoiding the use of organic solvents, minimizing hazardous waste streams, and using abundant materials (Sheldon \& Woodley, 2018). However, the adoption of biocatalytic methods relies on the development of scalable, economical, and operationally accessible reaction platforms. Recently, significant effort has been dedicated to establishing efficient biocatalytic reaction platforms for a wide variety of transformations. A number of approaches have been developed for conducting preparative-scale biocatalytic reactions, including reactions with purified enzymes, crude cell lysates (Rudroff et al., 2018; Sheldon \& Woodley, 2018) immobilized enzymes (Liang, Li, \& Yang, 2000; Sheldon \& van Pelt, 2013; Soares et al., 2011) lyophilized lysates, and wet whole cells (de Carvalho, 2017; Lin \& Tao, 2017; Wachtmeister, Mennicken, Hunold, \& Rother, 2016). Enzymes in various forms can be employed in batch or flow reactors (Britton, Majumdar, \& Weiss, 2018; Tamborini, Fernandes, Paradisi, $\&$ Molinari, 2018). Whole-cell (WC) transformations present several advantages over using isolated enzymes for in vitro reactions. For example, supply costs associated with the generation of WC preparations are much lower than for isolated proteins. Specifically, the cost of affinity chromatography resins, protein concentration devices, and cell lysis equipment is avoided for WC methods. WC preparations are also less time intensive, as laborious protein purification steps are not required. Similarly, crude cell lysate preparations also avoid a full protein purification, capturing some of these advantages. These attributes make WC biocatalysts more accessible to chemists and help promote the broader use of biocatalysts in organic synthesis. Capitalizing on these advantages, the use of WC biocatalysis for chemical synthesis has become increasingly prevalent in both academic and industrial settings (Bommarius, 2015; Bornscheuer et al., 2012; de Carvalho, 2017; Li et al., 2016; Parmeggiani, Weise, Ahmed, \& Turner, 2018; Schrittwieser, Velikogne, Hall, \& Kroutil, 2018; Wachtmeister \& Rother, 2016). Recently, Flitsch and Turner reported a WC biocatalytic process for the stereoselective amination of benzylic C-H bonds (Both et al., 2016). 
This feat was accomplished by co-transforming two plasmids containing genes corresponding to four enzymes necessary for a biocatalytic cascade (a monooxygenase, two alcohol dehydrogenases, and a $\omega$-transaminase, Figure 1b). By incubating the reaction components with the requisite biocatalysts in WC form in a sealed reaction vessel, they were able to produce chiral amines $(\mathbf{8})$ with moderate yields and high enantioselectivity. Without a WC biocatalytic platform, this process would have been logistically cumbersome, requiring the expression and potential isolation of each enzyme individually.

On an industrial scale, several processes have been designed to employ WC biocatalysts. Notably, WC biocatalysis has been utilized for the generation of valuable chemical feedstocks such as enantio-enriched alcohols, (Matsuyama, Yamamoto, \& Kobayashi, 2002) performance material monomers, (Kaluzna et al., 2016) and pharmaceutically relevant intermediates, (Guo et al., 2017) as well as the late-stage functionalization of active pharmaceutical ingredients during route development (Figure 1a; Guo et al., 2017; Milker, Fink, Rudroff, \& Mihovilovic, 2017; Xie \& Tang, 2007). Recently, researchers at GlaxoSmithKline demonstrated that a WC biocatalysis platform is also useful for discovery of new synthetic routes on the gram-scale, developing several biocatalytic routes to chiral 1,3-substituted cyclohexanones, such as 2 (Hadi et al., 2018). After obtaining initial hits from enzyme libraries, the use of WC biocatalysts allowed the
GlaxoSmithKline team to rapidly scale reactions and ultimately provide the desired chiral cyclohexanone products in gram quantities.

We previously established a platform for WC biocatalytic oxidative dearomatization of phenols using the flavin-dependent monooxygenase TropB (Baker Dockrey, Lukowski, Becker, \& Narayan, 2018). The native biosynthetic function of TropB was first characterized by Cox and coworkers in 2012 (Abood et al., 2015; Davison et al., 2012). TropB is an oxygenase, involved in the biosynthesis of stipitatic acid, that naturally converts 3-methyl-orcinaldehyde (9) to dienone $\mathbf{1 0}$ with perfect site- and stereoselectivity (Figure 2) (Abood et al., 2015; Baker Dockrey et al., 2018; Davison et al., 2012). We have previously demonstrated that TropB possesses a broad substrate scope and is able to generate a diverse array of dienone products (Baker Dockrey et al., 2018). The products generated through this powerful reaction can serve as building blocks to valuable bioactive natural products, including tropolones and azaphilones (Abood et al., 2015; Baker Dockrey et al., 2018; Davison et al., 2012; Gao, Yang, \& Qin, 2013). Over the last two decades, several chemical reagents have been developed to affect this difficult transformation (Bosset et al., 2014; Roche \& Porco, 2011; Volp \& Harned, 2013; Wu, Zhang, \& You, 2016; Zhu, Grigoriadis, Lee, \& Porco, 2005). Despite advances in synthetic oxidative dearomatization methodology, these

(a) Scaffolds accessed through WC biocatalysis

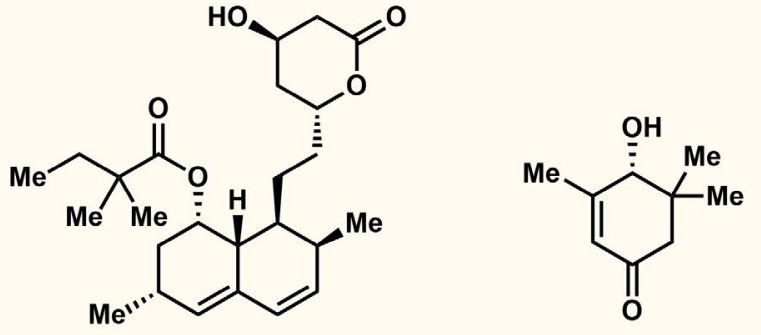

Simvastatin $\mathbf{1}^{[22]}$

(R)-4-hydroxyisophorone $2^{[19]}$<smiles>O[C@@H](CCl)c1ccc(F)c(F)c1</smiles>

Ticagrelor intermediate $\mathbf{3}^{[20]}$

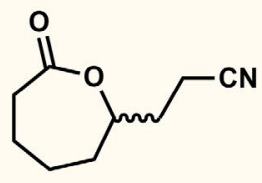

Nylon-9 monomer precursor $4^{[21]}$

(b) Benzylic amination enabled by WC biocatalytic enzyme cascade ${ }^{[17]}$

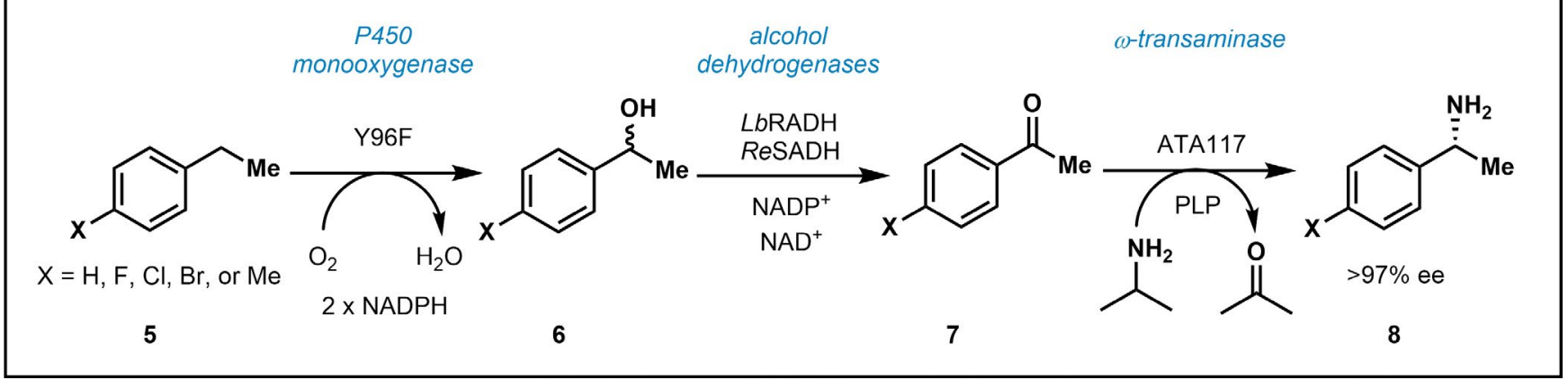

FI G URE 1 Examples of WC biocatalytic processes. (a) Select scaffolds accessed through WC biocatalytic reactions (Baker Dockrey et al., 2018; Guo et al., 2017; Hadi et al., 2018; Kaluzna et al., 2016; Matsuyama et al., 2002; Milker et al., 2017; Xie \& Tang, 2007). (b) WC biocatalysis utilizing a multi-enzyme cascade to generate chiral benzylic amines (Both et al., 2016) [Colour figure can be viewed at wileyonlinelibrary.com] 


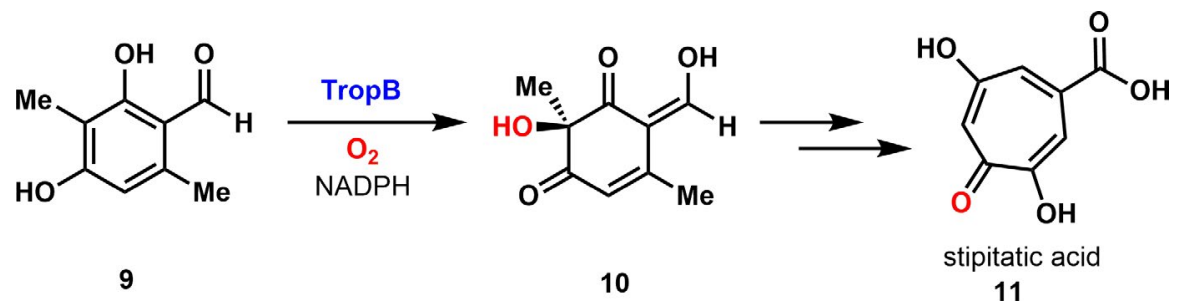

F I G U RE 2 Native reactivity of TropB within the stipitatic acid (11) biosynthetic pathway [Colour figure can be viewed at wileyonlinelibrary. com]

reactions often exhibit substrate-controlled site-selectivity, low levels of stereoselectivity, and can require stoichiometric chiral reagents. In contrast, Nature has conquered this reaction by using common biochemical cofactors and molecular oxygen, along with an evolved active site to provide impeccable site- and stereoselectivity. The advantages of biocatalytic oxidative dearomatization inspired our interest in developing TropB as a synthetically useful catalyst.

Flavin-dependent monooxygenases employ the noncovalent cofactor flavin adenine dinucleotide (FAD, 12), which following reduction to $\mathrm{FADH}_{2}(\mathbf{1 4})$ can react with molecular oxygen to form C4- $\alpha$-hydroperoxyflavin 15 (Figure 3) (Ullrich \& Hofrichter, 2007). This highly reactive species serves as an electrophilic source of oxygen for the oxidative dearomatization of electron-rich phenolic substrates. In the final step, loss of water from $\mathbf{1 7}$ regenerates FAD to close the catalytic cycle. We initially recognized that the requirement for stoichiometric NADPH is a potentially limiting factor in the scalability of this reaction. To address this limitation, we employed a well-established NADPH recycling system. Using this system, cofactor recycling could be successfully integrated into our WC reactions with native and non-native substrates on gram-scale to obtain complete conversion of substrates to dearomatized products (Baker Dockrey et al., 2018).

Our platform for WC reactions utilizes well-established biochemical tools to rapidly generate biocatalyst in high quantities (Figure 4). The general workflow begins with transformation of an expression plasmid containing the target gene, tropB, into E. coli BL21(DE3) cells, followed by overexpression of TropB in nutrient-rich media. After harvesting and flash-freezing the cells containing TropB, these cells can

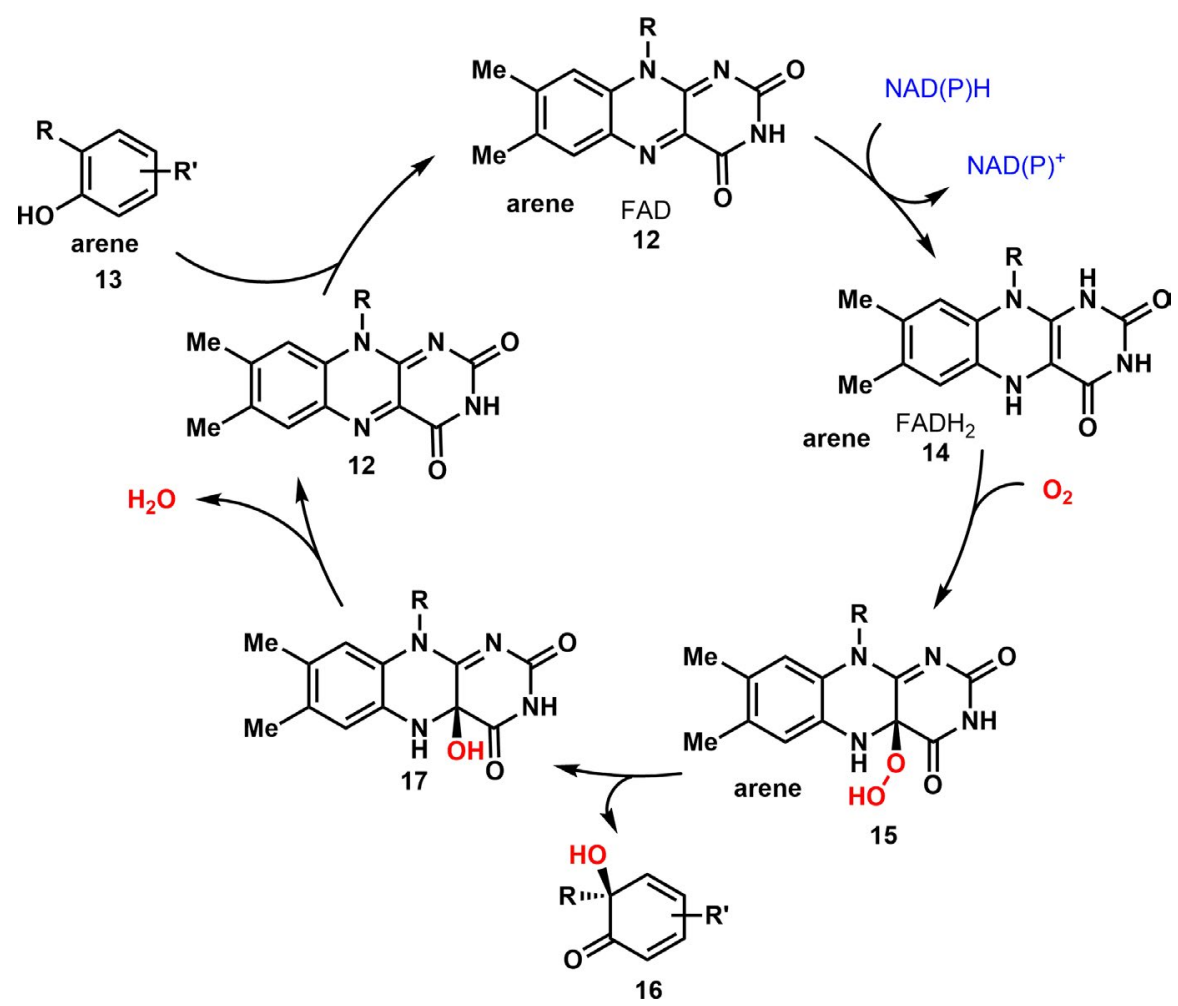

F I G URE 3 Proposed catalytic cycle of type A flavin-dependent monooxygenases in oxidative dearomatization of phenolic substrates (Ullrich $\&$ Hofrichter, 2007) [Colour figure can be viewed at wileyonlinelibrary.com] 


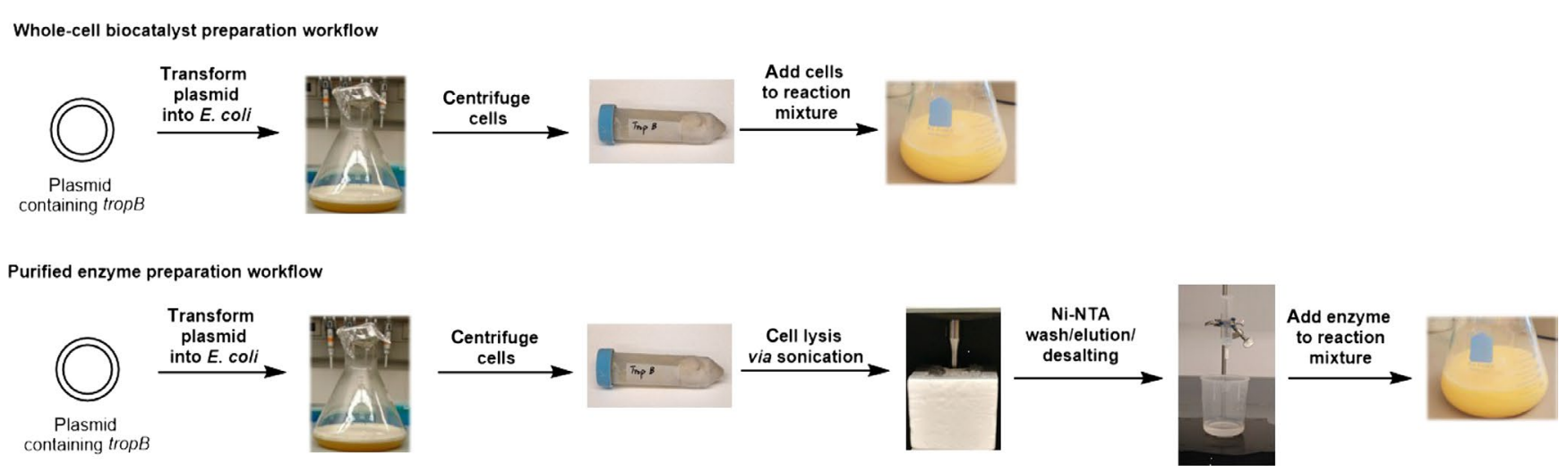

F I G U RE 4 Workflow of whole-cell reaction versus purified enzyme for TropB biocatalysis. Plasmid containing tropB is transformed into $E$. coli and then expressed. E. coli cells harboring TropB are harvested by centrifugation and frozen. The pellet can be added directly to the reaction flask. Additional steps are required to prepare purified protein [Colour figure can be viewed at wileyonlinelibrary.com]

be immediately used for oxidative dearomatization reactions. In contrast, obtaining protein for in vitro reactions requires several additional steps beyond protein expression, including cell lysis, purification by immobilized metal affinity chromatography, and desalting procedures. Thus, WC methods significantly decrease the time and resources required to prepare the catalyst for use in reactions.

The workflow employed for WC biocatalysis included the following steps. A gene, codon-optimized for heterologous expression in E. coli, was synthesized and cloned into pET151 by GeneArt (ThermoFisher). The plasmid DNA was transformed into chemically competent $E$. coli BL21(DE3) by standard heat-shock technique. An individual colony was selected and grown overnight in a 5-ml culture of LB media supplemented with ampicillin for plasmid maintenance. A 500-ml culture of sterilized Terrific Broth was supplemented with ampicillin and inoculated with $5 \mathrm{ml}$ of overnight culture. This culture was incubated at $37^{\circ} \mathrm{C}$ until the cell density $\left(\mathrm{OD}_{600}\right)$ reached $0.6-0.8$, at which point the culture was cooled to $20^{\circ} \mathrm{C}$ and protein overexpression was induced by addition of IPTG $(0.1 \mathrm{mM}$ final concentration). The culture was incubated at $20^{\circ} \mathrm{C}$ for $14-16 \mathrm{hr}$ before harvesting the cells via centrifugation $(4,000 \times g$ for $20 \mathrm{~min})$.

Two methods were used to prepare the cell pellet for use in WC reactions: flash-freezing or lyophilization. For the former, the crude cell pellet was flash frozen in liquid nitrogen in small aliquots. This method greatly alleviated difficulties in weighing out wet cell pellet for accurate quantification of our WC reactions. For the preparation of lyophilized cells, the crude cell pellet was aliquoted into 1 gram portions and resuspended in an excipient solution $(0.5 \mathrm{~L}, 10 \% \mathrm{w} / \mathrm{v}$ excipient in Milli-Q water) and pelleted in several $50 \mathrm{ml}$ Falcon tubes. The tubes were then covered with a KimWipe and lyophilized for 48-72 hr. To identify the ideal preparation method for lyophilized cell pellet for use in our WC reactions, we added various excipients to cells prior to lyophilization based upon a survey of common excipients used in the literature (Baheti,
Kumar, \& Bansal, 2010; Bedu-Addu, 2004; Randolph, 1997). We then compared the activity of each preparation in $1 \mathrm{~g}$ scale reactions with the non-native substrate $\mathbf{1 8}$ (Table 1). The results of these experiments indicated that enzyme activity was maintained in lyophilized cells, and only improved nominally by the addition of $10 \mathrm{wt} \%$ sucrose or PEG 4000 (entries 3 and 4, respectively) (Baheti et al., 2010; Bedu-Addu, 2004; Randolph, 1997). This method simplified the handling of the biocatalytic reagent, streamlining the set-up of large-scale reactions.

Preparative-scale WC enzymatic reactions were conducted on $1 \mathrm{~g}$ of substrate under the following conditions: 20 weight equivalents of wet cell pellet, $5 \mathrm{mM}$ substrate, $10 \%$ (v/v) toluene (caution: highly flammable solvent), $0.1 \mathrm{mM}$ $\mathrm{NADP}^{+}, \quad 0.1 \mathrm{U} / \mathrm{ml}$ glucose-6-phosphate dehydrogenase (G6PDH), and $10 \mathrm{mM}$ glucose-6-phosphate for NADPH generation in reaction buffer $(50 \mathrm{mM}$ potassium phosphate buffer, $\mathrm{pH}$ 8.0). The reaction components were combined in a $1 \mathrm{~L}$ Erlenmeyer flask and incubated at $30^{\circ} \mathrm{C}$ for $2 \mathrm{hr}$ at $100 \mathrm{rpm}$. The reaction mixture was filtered through Celite, acidified to $\mathrm{pH} 2.0$ and extracted with ethyl acetate $(3 \times 500 \mathrm{ml})$. The

TA B L E 1 Optimization of gram-scale reactions with lyophilized cell pellet

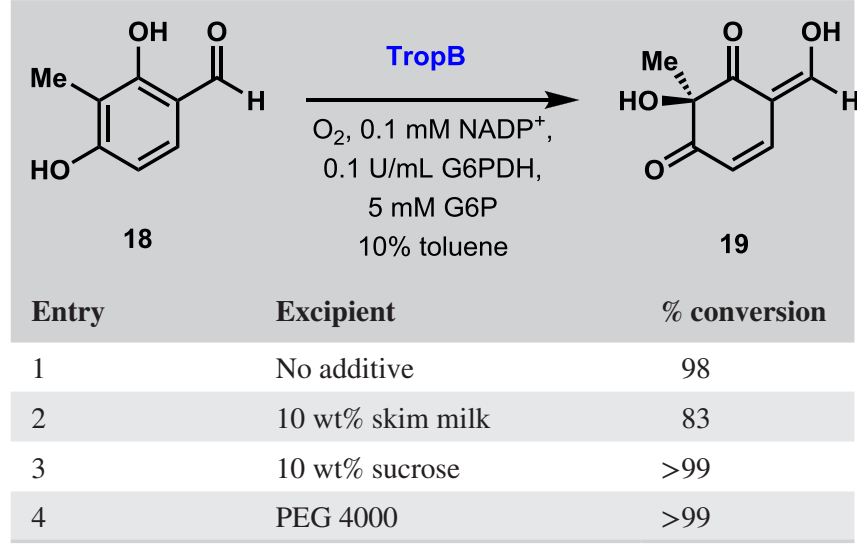



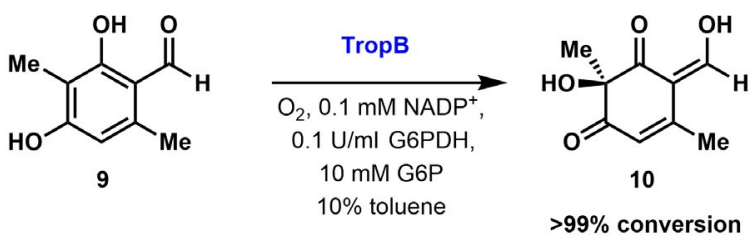

$56 \%$ isolated yield

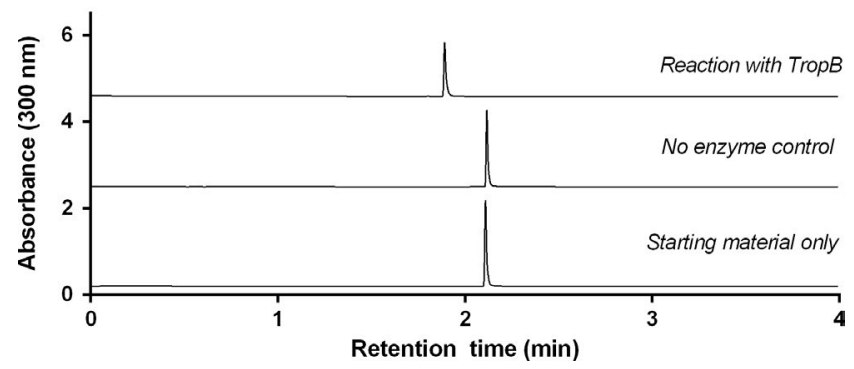

F I G URE 5 UPLC trace for oxidative dearomatization of 9 by TropB [Colour figure can be viewed at wileyonlinelibrary.com]

combined organic layers were dried over sodium sulfate, filtered and concentrated under reduced pressure. The resulting mixture was purified on silica gel (methanol/acetic acid/dichloromethane, 1:1:10) to afford the $o$-quinol product. For smaller scale reactions $(5 \mathrm{mg})$, the same procedure was employed, except a $100-\mathrm{ml}$ Erlenmeyer flask was used in place of a $1 \mathrm{~L}$ flask. To monitor reaction progress, $50 \mu \mathrm{l}$ aliquots were removed from the reaction and quenched with $75 \mu \mathrm{l}$ of methanol supplemented with an internal standard, $2.5 \mathrm{mM}$ pentamethyl benzene. Reaction conversion was monitored by UPLC-DAD, and starting material consumption was quantified by comparison with a substrate calibration curve (Figure 5).

\section{\begin{tabular}{l|l}
2 & RESULTS AND DISCUSSION
\end{tabular}}

Whole-cell reactions with TropB were initially performed in aqueous buffer alone. Under these conditions, conversions of starting material were low, which we hypothesized was due to poor substrate solubility in the aqueous reaction environment. To resolve this issue, we tested the effect of both water-miscible and immiscible solvents on the WC reaction (Table 2). Toluene improved conversion to dienone 10, with full conversion in $2 \mathrm{hr}$ under the described conditions. Additionally, we screened reactions with a reduced amount of wet cells and noted complete conversion with cell loading as low as 20 weight equivalents. These optimized conditions were applied to gram-scale reactions, further demonstrating the appeal of this method for synthetic applications.

Using our optimized WC biocatalysis platform, the overall yield of usable biocatalyst from each protein expression was significantly higher than what was obtained following protein purification for in vitro reactions. In this case, more than $20 \mathrm{WC}$ preparative-scale $(100 \mathrm{mg}$ ) reactions could be
T A B L E 2 Optimization of WC TropB reaction with co-solvents

\begin{tabular}{|lllc|}
\hline Entry & $\begin{array}{l}\text { Cell pellet } \\
(\mathbf{m g})\end{array}$ & $\begin{array}{l}\text { Co-solvent }(\mathbf{1 0} \% \\
\mathbf{v} / \mathbf{v})\end{array}$ & $\begin{array}{l}\text { \% conversion } \\
\text { to } \mathbf{1 0}\end{array}$ \\
\hline 1 & 500 & No co-solvent & 23 \\
\hline 2 & 500 & Ethanol & 54 \\
\hline 3 & 500 & Acetonitrile & $<5$ \\
\hline 4 & 500 & Methanol & 62 \\
\hline 5 & 500 & Tetrahydrofuran & $<5$ \\
\hline 6 & 500 & Toluene & $>99$ \\
\hline 7 & 100 & Toluene & $>99$ \\
\hline 8 & 50 & Toluene & 86 \\
\hline
\end{tabular}

T A B L E 3 Scalability of WC platform compared to in vitro platform

\begin{tabular}{|lll|} 
Platform & Scale of reactions $(\mathbf{m g})$ & $\begin{array}{l}\text { Reactions per } \\
\text { liter cell culture }\end{array}$ \\
\hline In vitro & 100 & $2-3$ \\
\hline WC & 100 & $>20$ \\
\hline
\end{tabular}

carried out for every liter of TropB expression cell culture. In comparison, from $1 \mathrm{~L}$ of expression culture, the quantity of purified protein was sufficient for 2-3 reactions on the same scale (Table 3 ). We suspect this was due to a loss of protein and activity over the course of the purification procedure. Reactions carried out with crude cell lysate gave diminished conversions relative to both in vitro and WC reactions, indicating that some enzyme stabilization may have been provided by the cellular environment. The WC biocatalyst also required significantly less time to prepare, saving 5-6 hr for each batch of catalyst. Therefore, our WC platform demonstrated superior efficiency over in vitro reactions in both preparation time and scalability of the reaction.

\section{3 | CONCLUSIONS}

In summary, we have developed a scalable and economical WC biocatalytic method for oxidative dearomatization of phenols. This biocatalytic method improves upon previously developed chemical methods in terms of environmental sustainability, as it uses water as a solvent and a renewable biological catalyst as the oxidant. In comparison with in vitro reactions, WC biocatalytic methods can provide distinct and significant advantages. With no requirement for enzyme purification, WC procedures reduce the overall time, cost and specialized equipment associated with catalyst production. After the simple optimization of several parameters, such as cell preparation method and reaction co-solvent, our WC platform allowed us to perform tenfold more reactions per liter of cell culture when 
compared to purified enzyme. This improvement was achieved without loss of catalytic function or selectivity. Moreover, this highly scalable method provides a simple and accessible reaction platform, promoting use of biocatalysts in synthetic chemistry.

\section{ACKNOWLEDGMENTS}

This work was supported by funds from the University of Michigan Life Sciences Institute and the National Institutes of Health R35 GM124880 (to A.R.H.N.). S.B.D. is supported by the Chemical-Biology Interface Training Program at the University of Michigan. T.J.D. thanks the National Science Foundation for a Graduate Research Fellowship.

\section{CONFLICT OF INTEREST}

The authors declare no conflicts of interest.

\section{ORCID}

Alison R. H. Narayan (D) http://orcid.org/0000-0001-8290-0077

\section{REFERENCES}

Abood, A., Al-Fahad, A., Scott, A., Hosny, A. E.-D. M. S., Hashem, A. M., Fattah, A. M. A., ... Cox, R. J. (2015). Kinetic characterisation of the FAD dependent monooxygenase TropB and investigation of its biotransformation potential. RSC Advances, 5, 49987-49995.

Baheti, A., Kumar, L., \& Bansal, A. K. (2010). Excipients used in lyophilization of small molecules. Journal of Excipients and Food Chemicals, 41, 41-54.

Baker Dockrey, S. A., Lukowski, A. L., Becker, M. R., \& Narayan, A. R. H. (2018). Biocatalytic site- and enantioselective oxidative dearomatization of phenols. Nature Chemistry, 10, 119.

Bedu-Addu, F. K. (2004). Understanding lyophilization formulation

development. Pharmaceutical Technology (Lyophilization), 2004(2), 10-18.

Bommarius, A. S. (2015). Biocatalysis: A status report. Annual Review of Chemical and Biomolecular Engineering, 6, 319-345.

Bornscheuer, U. T., Huisman, G. W., Kazlauskas, R. J., Lutz, S., Moore, J. C., \& Robins, K. (2012). Engineering the third wave of biocatalysis.. Nature, 485, 185.

Bosset, C., Coffinier, R., Peixoto, P. A., El Assal, M., Miqueu, K., Sotiropoulos, J. M., ... Quideau, S. (2014). Asymmetric hydroxylative phenol dearomatization promoted by chiral binaphthylic and biphenylic iodanes. Angewandte Chemie (International ed. in English), 53, 9860-9864.

Both, P., Busch, H., Kelly, P. P., Mutti, F. G., Turner, N. J., \& Flitsch, S. L. (2016). Whole-cell biocatalysis for stereoselective C-H amination reactions. Angewandte Chemie International Edition, 55, 1511-1513.

Britton, J., Majumdar, S., \& Weiss, G. A. (2018). Continuous flow biocatalysis. Chemical Society Reviews, 47, 5891-5918.

de Carvalho, C. C. (2017). Whole cell biocatalysts: Essential workers from Nature to the industry. Microbial Biotechnology, 10, 250-263.
Davison, J., Al Fahad, A., Cai, M., Song, Z., Yehia, S. Y., Lazarus, C. M., ... Cox, R. J. (2012). Genetic, molecular, and biochemical basis of fungal tropolone biosynthesis. Proceedings of the National Academy of Sciences of the United States of America, 109, 7642-7647.

Gao, J. M., Yang, S. X., \& Qin, J. C. (2013). Azaphilones: Chemistry and biology. Chemical Reviews, 113, 4755-4811.

Guo, X., Tang, J.-W., Yang, J.-T., Ni, G.-W., Zhang, F.-L., \& Chen, S.-X. (2017). Development of a practical enzymatic process for preparation of (S)-2-Chloro-1-(3,4-difluorophenyl)ethanol. Organic Process Research \& Development, 21, 1595-1601.

Hadi, T., Diaz-Rodríguez, A., Khan, D., Morrison, J. P., Kaplan, J. M., Gallagher, K. T., ... Roiban, G.-D. (2018). Identification and implementation of biocatalytic transformations in route discovery: Synthesis of chiral 1,3-substituted cyclohexanone building blocks. Organic Process Research \& Development, 22(7), 871-879.

Kaluzna, I., Schmitges, T., Straatman, H., van Tegelen, D., Müller, M., Schürmann, M., \& Mink, D. (2016). Enabling selective and sustainable p450 oxygenation technology. production of 4-hydroxy- $\alpha$-isophorone on kilogram scale. Organic Process Research \& Development, 20, 814-819.

Li, A., Ilie, A., Sun, Z., Lonsdale, R., Xu, J.-H., \& Reetz, M. T. (2016). Whole-cell catalyzed multiple regio- and stereoselective functionalizations in cascade reactions enabled by directed evolution. Angewandte Chemie International Edition, 55, 12026-12029.

Liang, J. F., Li, Y. T., \& Yang, V. C. (2000). Biomedical application of immobilized enzymes. Journal of Pharmaceutical Sciences, 89 , 979-990.

Lin, B., \& Tao, Y. (2017). Whole-cell biocatalysts by design. Microbial Cell Factories, 16, 106.

Matsuyama, A., Yamamoto, H., \& Kobayashi, Y. (2002). Practical application of recombinant whole-cell biocatalysts for the manufacturing of pharmaceutical intermediates such as chiral alcohols. Organic Process Research \& Development, 6, 558-561.

Milker, S., Fink, M. J., Rudroff, F., \& Mihovilovic, M. D. (2017). Nonhazardous biocatalytic oxidation in Nylon-9 monomer synthesis on a $40 \mathrm{~g}$ scale with efficient downstream processing. Biotechnology and Bioengineering, 114, 1670-1678.

Parmeggiani, F., Weise, N. J., Ahmed, S. T., \& Turner, N. J. (2018). Synthetic and therapeutic applications of ammonia-lyases and aminomutases. Chemical Reviews, 118, 73-118.

Randolph, T. W. (1997). Phase separation of excipients during lyophilization: Effects on protein stability. Journal of Pharmaceutical Sciences, 86, 1198-1203.

Roche, S. P., \& Porco Jr, J. A. (2011). Dearomatization strategies in the synthesis of complex natural products. Angewandte Chemie (International ed. in English), 50, 4068-4093.

Rudroff, F., Mihovilovic, M. D., Gröger, H., Snajdrova, R., Iding, H., \& Bornscheuer, U. T. (2018). Opportunities and challenges for combining chemo- and biocatalysis. Nature Catalysis, 1, 12-22.

Schrittwieser, J. H., Velikogne, S., Hall, M., \& Kroutil, W. (2018). Artificial biocatalytic linear cascades for preparation of organic molecules. Chemical Reviews, 118, 270-348.

Sheldon, R. A., \& van Pelt, S. (2013). Enzyme immobilisation in biocatalysis: Why, what and how. Chemical Society Reviews, 42, 6223-6235.

Sheldon, R. A., \& Woodley, J. M. (2018). Role of biocatalysis in sustainable chemistry. Chemical Reviews, 118, 801-838.

Soares, J. C., Moreira, P. R., Queiroga, A. C., Morgado, J., Malcata, F. X., \& Pintado, M. E. (2011). Application of immobilized enzyme 
technologies for the textile industry: A review. Biocatalysis and Biotransformation, 29, 223-237.

Tamborini, L., Fernandes, P., Paradisi, F., \& Molinari, F. (2018). Flow bioreactors as complementary tools for biocatalytic process intensification. Trends in Biotechnology, 36, 73-88.

Ullrich, R., \& Hofrichter, M. (2007). Enzymatic hydroxylation of aromatic compounds.. Cellular and Molecular Life Sciences, 64, 271-293.

Volp, K. A., \& Harned, A. M. (2013). Chiral aryl iodide catalysts for the enantioselective synthesis of para-quinols. Chemical Communications (Cambridge, England), 49, 3001-3003.

Wachtmeister, J., Mennicken, P., Hunold, A., \& Rother, D. (2016). Modularized biocatalysis: Immobilization of whole cells for preparative applications in microaqueous organic solvents. ChemCatChem, 8, 607-614.

Wachtmeister, J., \& Rother, D. (2016). Recent advances in whole cell biocatalysis techniques bridging from investigative to industrial scale. Current Opinion in Biotechnology, 42, 169-177.

Wu, W. T., Zhang, L., \& You, S. L. (2016). Catalytic asymmetric dearomatization (CADA) reactions of phenol and aniline derivatives. Chemical Society Reviews, 45, 1570-1580.
Xie, X., \& Tang, Y. (2007). Efficient synthesis of simvastatin by use of whole-cell biocatalysis. Applied and Environment Microbiology, 73, 2054-2060.

Zhu, J., Grigoriadis, N. P., Lee, J. P., \& Porco, J. A. (2005). Synthesis of the azaphilones using copper-mediated enantioselective oxidative dearomatization. Journal of the American Chemical Society, 127, 9342-9343.

How to cite this article: Baker Dockrey SA, Doyon TJ, Perkins JC, Narayan ARH. Whole-cell biocatalysis platform for gram-scale oxidative dearomatization of phenols. Chem Biol Drug Des. 2019;93:1207-1213. https://doi.org/10.1111/cbdd.13443 